\title{
Update on the listeriosis outbreak
}

L ate this summer, an outbreak of listeriosis sickened more than 130 individuals in 26 states. The infection, caused by the bacteria Listeria monocytogenes, was eventually linked to contaminated cantaloupe, and the outbreak has been blamed for at least 26 deaths. The JCI recently spoke to Martin Wiedmann, professor of Food Science at Cornell University, about this outbreak and about food safety more generally.

JCI: How was Listeria introduced into the food supply?

Wiedmann: Listeria monocytogenes can be introduced into the food supply at any point of the farm-to-table continuum. Stringent food safety measures have played a key role in reducing the prevalence of $L$. monocytogenes in ready-to-eat foods, such as deli meats. For foods that do not undergo any treatments that inactivate L. monocytogenes, contamination in any point of the food chain can be carried through to the final consumed product. Importantly, unlike for enterohemorrhagic E. coli and Salmonella, extremely high numbers of L. monocytogenes are required in the contaminated food that is consumed to lead to infection of the consuming host. Hence, only food products that will allow growth of L. monocytogenes after contamination are likely to cause human cases or outbreaks. In addition, only certain subpopulations (the elderly, pregnant women, and the immunocompromised) are likely to contract listeriosis.

While the specific source of L. monocytogenes in the recent outbreak linked to consumption of contaminated cantaloupe has not yet been definitively identified, it appears likely that the main sources responsible for contamination of the cantaloupes were in the environment of the facility that was used to wash, pack, and store them after harvest.

JCI: Why are listeriosis outbreaks so deadly?

Wiedmann: While L. monocytogenes infections can lead to mild flu-like symptoms and, in rare cases, gastrointestinal disease symptoms, typical listeriosis cases present as an invasive disease with symptoms including septicemia, meningitis, and spontaneous abortions in pregnant women. While invasive listeriosis represents a rare foodborne disease (with 3-5 cases of listeriosis per year per million population being a typical rate for most developed countries), a large proportion of the cases (approximately 20\%) have a lethal outcome. By comparison, approximately $0.5 \%$ of both E. coli $\mathrm{O} 157$ and salmonellosis cases

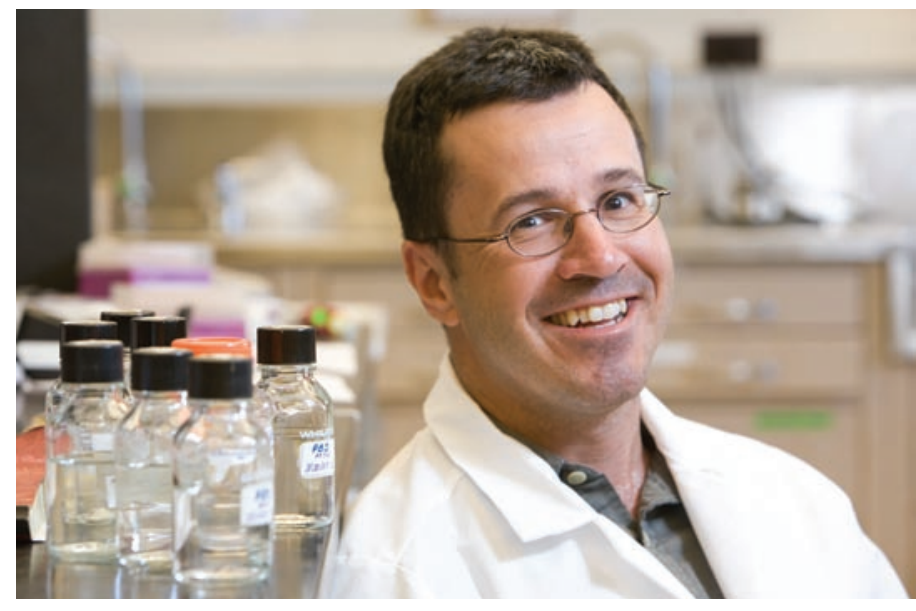

lead to deaths of the infected individual. From a mechanistic point of view, $L$. monocytogenes infections are characterized by this pathogen's ability not to only invade and survive in host cells, but also to spread from host cell to host cell without exiting in the extracellular space, which facilitates avoidance of the host's humoral immune system. In addition, L. monocytogenes has the ability to penetrate the intestinal, placental, and blood-brain barriers.

JCI: How common are foodborne illnesses? Wiedmann: In the United States, approximately 48 million cases of gastrointestinal, domestically acquired foodborne illnesses occur annually. These estimates indicate that 1 of 6 Americans experiences a foodborne illness episode annually.

Globally, it was reported that in 2005 alone, 1.8 million people died from diarrheal diseases, with many of these cases likely due to contaminated food and drinking water.

JCI: How are outbreaks traced back to a specific source?

Wiedmann: Outbreak traceback really includes two separate, but often overlapping efforts: detection of a putative outbreak and traceback of that putative outbreak to a source. Outbreaks are typically detected by either an increased number of cases of a given disease above the baseline or by identification of multiple cases that are linked through molecular subtyping data. In the listeriosis outbreak linked to cantaloupe, the outbreak was initially detected by public health officials in Colorado, who observed a much larger number of human listeriosis cases over the course of a few weeks than are typical. State health departments through- out the U.S. perform molecular subtyping of human disease-associated isolates for key foodborne pathogens and submit them to the CDC-led PulseNet system, allowing for comparison of subtype data across the U.S. and identification of multiple cases caused by the same subtype. After identification of a linked cluster of cases, epidemiological investigations using food consumption questionnaires and subsequent case-control studies are used to identify foods for which consumption is significantly overrepresented among outbreak cases. Definitive identification of an outbreak often involves isolation of the pathogen subtype from the food that has been implicated as the outbreak source in an epidemiological investigation.

JCI: Should outbreaks like this change the way the average person selects or handles food on a daily basis?

Wiedmann: For some foodborne disease outbreaks, consumer practices or precautions will only achieve limited reductions in the likelihood of disease. However, every outbreak must serve as a reminder to consumers that they play an important role in reducing the risk of foodborne illnesses. Regarding this most recent outbreak, specific CDC recommendations for consumers consuming melons have been detailed (1).

\section{Kathryn Claiborn}

1. Centers for Disease Control and Prevention. Investigation Update: Multistate Outbreak of Listeriosis Linked to Whole Cantaloupes from Jensen Farms, Colorado. CDC Web site. http://www. cdc.gov/listeria/outbreaks/cantaloupes-jensenfarms/092711/index.html. Updated September 26, 2011. Accessed November 3, 2011. 